\title{
Commentary
}

\section{How can nurses best help women facing breast cancer?}

\author{
Dennis L Citrin* and Jessica Kapustin \\ Cancer Treatment Centers of America at Midwestern Regional Medical Center, Zion Illinois, USA
}

One in eight American women will be diagnosed with breast cancer during her lifetime. According to the American Cancer Society's estimates for breast cancer in women, 231,840 new cases of invasive breast cancer and 62,290 new cases of carcinoma in situ (DCIS) will be diagnosed in the United States in the year 2015 [1]. That means approximately 5,656 women will be diagnosed with breast cancer every week. Most health care professionals will have some interaction with breast cancer patients during their professional lives.

It's no exaggeration to say that there has been an explosion in our knowledge regarding the biology of breast cancer over the past decade or so, and this has led to major improvements in how we treat breast cancer patients.

There have been advances in all of the major treatment disciplines used in breast cancer management including surgery, radiation therapy and medical oncology. Modern breast cancer treatment is not only much more effective than previously, but it is also much less likely to cause major side-effects and is better tolerated by the patient [2].

These facts, combined with better diagnostic methods and the proven value of screening mammography leading to earlier diagnosis and lowered death rates, allow us to be much more optimistic and reassuring for women newly diagnosed with early stage breast cancer.

It's not all good news, however. The unfortunate fact is that many women are still being denied the benefits of modern breast cancer diagnosis and treatment. In this article we will discuss the reasons for this, and also suggest ways nurses can help improve the situation and best serve women facing breast cancer.

Our breast cancer accredited center of excellence is located within a cancer hospital in northern Illinois. We work as part of a multidisciplinary team, which includes surgical oncologists, radiation and medical oncologists, pathologists, diagnostic radiologists, nurse practitioners, nurse navigators, surgical nurses, naturopathic physicians, nutritionists, mind body specialists and oncology rehabilitation specialists. Each year our team sees hundreds of new patients with breast cancer.

Why do we claim that many women do not benefit as much as they should from modern breast cancer diagnosis and treatment? There are two major reasons: first, despite all of the major publicity regarding the importance of early diagnosis, many women still delay reporting a breast lump to their doctors [3]. Second a significant number of women with breast cancer do not complete the treatment course recommended by their doctors [4].

\section{Patient delay}

Our team recently reviewed the medical records of patients with breast cancer seen during a twelve month period. During this year, 591 women with different stages and types of breast cancer were examined.
In 95 of these 591 women (16\%), their initial diagnosis was delayed at least six months.

What's worse, the average delay between when the patient first felt a lump or abnormality to when she was correctly diagnosed was twelve months. Fourteen patients waited two years or longer before seeing a doctor. As a result, by the time they came to us, many of the patients had advanced disease that was much more difficult to treat effectively [3].

A serious medical condition like cancer should be diagnosed at the earliest possible moment and at the earliest possible stage. So what's causing the delay in diagnosis?

In 66 of the 591 patients (11.3 percent of the total), the delay was caused by the patients themselves. They chose not to seek the advice of doctors even though each had felt a suspicious lump in their breast. Their lumps persisted and grew in size before these women finally sought medical help.

Twenty three of the 66 patients who delayed seeking medical help tried to treat their breast cancers with a variety of natural means, including taking herbs and supplements and changing their diets. In those patients, the delay in receiving appropriate cancer treatment was even longer, averaging 18 months.

The patient was not responsible for the delay in every case. Twenty seven women ( 4.5 percent of the total) went to see their doctors complaining of a lump and were incorrectly reassured by the doctor that they were "fine," either based on the doctor's examination alone, or because the mammogram and sometimes an ultrasound examination didn't suggest cancer. No biopsies were recommended.

Two women had delays in diagnoses because of lack of access to medical care, one was a victim of Hurricane Katrina, and the other didn't have health insurance.

What these numbers show is that in our patient population, approximately 1 of every 10 women who felt a potentially cancerous lump in her breast suspected that she had cancer, and yet waited an entire year on average before seeking appropriate medical care.

\section{Non-compliance with treatment recommendations}

We also conducted a second study of patients with recurrent breast cancer. These were patients who had been treated for early stage breast

Correspondence to: Dennis L Citrin, MD $\mathrm{PhD}$, Medical Oncologist, Cancer Treatment Centers of America at Midwestern Regional Medical Center, Zion Illinois, 60099, USA, Tel: 866-697-2224; E-mail: Dennis.Citrin@ctca-hope.com

Received: February 06, 2016; Accepted: March 05, 2016; Published: March 09, 2016 
cancer in other institutions, usually community hospitals, and were then referred to our specialized cancer treatment hospital when they experienced a relapse or recurrence of their disease.

We were interested to learn how many of these patients whose initial treatment was not successful in curing them, had completed all of the treatment recommended by their treating physicians.

Over a three year period, we saw 383 patients with recurrent breast cancer. 222 patients $(58 \%)$ completed all of the treatment recommended by their physicians, while 161 patients (42\%) did not. Eleven patients declined the recommended surgery; while 66 of the 299 patients $(23.8 \%)$ refused the recommended radiation therapy. 354 patients received appropriate adjuvant chemotherapy. 74 (20.9\%) either declined or failed to complete the recommended course of postoperative chemotherapy. Nearly forty percent of patients (102/261) either declined or terminated hormone treatment before receiving the recommended five years of therapy [5].

This study showed that patients are more likely to refuse recommended drug treatment than surgery or radiation, and that refusal of treatment is associated with an increased risk of treatment failure which is defined as recurrence of disease.

\section{Conclusions}

Taken together, these studies demonstrate that a significant number of American women continue to receive sub-optimal treatment for breast cancer. Whether they delay seeking diagnosis and prompt treatment, or refuse potentially life-saving treatment, they are reducing their chances of cure.

We believe the main reason for both delay and non-compliance with treatment recommendations is fear. Fear of cancer is very natural, and is based on decades of human experience. Cancer has been associated for centuries with the idea of suffering and inevitable death. Many patients are also very fearful of cancer treatment. In the past cancer treatments were relatively ineffective and often caused severe side-effects.

However, these perceptions are no longer true. Most women diagnosed with breast cancer today can confidently expect to be cured. Presently the 5 year survival rate for patients with with localized disease is $96.8 \%$ [6].

Not only is breast cancer treatment much more effective, it is also much less likely to cause permanent side-effects or disability. For example, radical mastectomy, a procedure where the entire breast and underlying muscle is removed, is rarely performed today. Most patients can be treated with breast-conserving surgery and many may not require even a simple mastectomy [2].

Another example includes drug treatment. Not only are modern breast cancer drugs much more effective, they are also much less likely to cause severe side-effects. Thirty years ago chemotherapy frequently caused severe nausea and vomiting. Today we have very effective antiemetics, which target the vomiting center in the brain. As a result, breast cancer patients receiving chemotherapy rarely experience severe or uncontrolled vomiting [7].

\section{The role of nurses in the care of the breast cancer patient}

If fear, based on inaccurate, outdated or misleading information, is the main factor, that deters women from seeking optimal breast cancer diagnosis and treatment, the best way to overcome that fear is with patient education.
Appropriate patient education is particularly important when dealing with a chronic life-threatening disease like breast cancer, where optimal treatment often requires the complex interaction of multiple disciplines and treatment can extend over many months; and in some cases years [8].

Nurses are uniquely positioned to provide that education on an ongoing basis. Doctors frequently do not have the time to fully address the patient's concerns, and too often doctors use medical jargon that patients just don't understand.

A July 2010 Wall Street Journal article described a study from the Centers for Disease Control and Prevention that reported that nearly 9 out of 10 adults had difficulty following medical advice because it was largely incomprehensible to them [9]. Numerous studies have shown that patients who do not fully understand what their doctors tell them are much more likely to skip necessary medical tests or fail to take medications properly, with much poorer outcomes as a result $[10,11]$. It has also been noted that at times patients are afraid to ask their doctors questions, because they are afraid their questions may be seen as stupid or silly.

Nurses consistently rate highest in honesty and ethical standards in Gallup surveys and it is believed that generally speaking, nurses are more likely to be perceived as empathetic and inspire patient confidence considering the results of this study [3].

We interviewed thirty patients who came to our institution with locally advanced or metastatic breast cancer after refusing conventional treatments. We wanted to understand what had motivated them to refuse treatment for what was in many cases very obvious progressive breast cancer.

What was revealed during these interviews was a startling conclusion. Almost all of the 30 patients in our study who refused conventional treatment felt rejected or disrespected by their original physicians after they asked about the possibility of using alternative medicine to treat their cancer.

When questioned by staff the patients stated that when they expressed an interest in alternative therapies, their doctors became angry or hostile and expressed very negative statements, such as, "If you do this, you'll never see your children grow up."

As a result of their doctors' negative reactions, they rejected all conventional cancer treatment (surgery, radiation and chemotherapy), and instead embraced a variety of natural treatments that, by themselves were incapable of preventing the progression of their disease. In all thirty patients that decision resulted in potentially curable cancers progressing to an advanced stage, when it was no longer curable [3].

It is clear that negative comments by health care professionals can have a serious effect on patients' feelings of self-respect, and can lead to their making inappropriate treatment choices.

Nurses can play a critically important role in counteracting negative comments, and play a very positive role in educating and supporting women with breast cancer throughout what is often a long, physically and emotionally stressful journey through the health care system.

\section{Breast cancer myths and facts}

To perform this role successfully however, the nurse has to be knowledgeable regarding the scientific basis and details of modern breast cancer treatment. At the same time understanding what their 
patients believe that science to be. These are not always the same, by any means.

There is a need for nursing specialists to care for and educate women with breast cancer during all phases of their diagnosis and treatment which will extend beyond active treatment to rehabilitation and survivorship.

One of the benefits of the internet is that there is a vast amount of information available to women about breast cancer, but this includes a tremendous amount of misinformation.

Some of the myths that we regularly hear regarding breast cancer include $[3,11,12]$ :

- Breast cancer is caused by any one of the following: Stress, Injury to the breast, too much sugar in your diet, hair dye, breast implants

- You can cure yourself of breast cancer naturally (by changing your diet, drinking alkaline water, macrobiotics, Vitamin C, homeopathics, Vitamin B17)

- Surgery will help a cancer spread

- A mammogram can cause breast cancer to spread

- Breast cancer is an incurable disease

- If you have a family history of breast cancer you are likely to develop breast cancer too

- Antiperspirants and deodorants cause breast cancer

- If I have a double mastectomy I'll never have to worry about getting breast cancer again

It's here that an informed compassionate nurse can best help his or her patients. Educating women about breast cancer, dispelling myths, addressing patients' natural fears and guiding them to make appropriate choices will greatly enhance their chances of being cured of breast cancer.

Nurses have a vital role in educating about prevention and health promotion and need to stress the importance of healthy lifestyles as well as the importance of early detection and early interventions.

Nurses have the ability to be active throughout a person's life. In building relationships with patients in various situations including health fairs, school events, community events and professional capacities nurses are able to educate on the importance of self-breast exams, clinical breast exams, and screening tests to assist in the diagnoses of breast cancer.

Once a patient is diagnosed the nurse has the ability to offer education along with physicians that can ensure that the patient is able to make a well informed educated decision regarding their plan of care.

During treatments, nurses and patients often build a unique relationship. Nurses are able to detect side effects of treatments early and with early intervention can decrease patients' symptoms thus increasing their quality of life.

Nurses remain active throughout patients' lifespans: encouraging, coaching and being the cheerleaders that empower patients to remain educated and compliant with screening recommendations, surveillance standards and the importance of self-care.

\section{References}

1. Breast Cancer Facts and Figures (2015)American Cancer Society 1-3.

2. Zurrida S, Veronesi U (2015) Milestones in breast cancer treatment. Breast J 21: 3-12. [Crossref]

3. Citrin DL, Bloom DL, Grutsch JF, Mortensen SJ, Lis CG (2012) Beliefs and perceptions of women with newly diagnosed breast cancer who refused conventional treatment in favor of alternative therapies. Oncologist 17: 607-612.[Crossref]

4. Citrin DL, Neelam R, Grutsch JF, Mortensen SJ (2011) Relationship between lack of patient compliance with adjuvant therapy guidelines and recurrence in early breast. $J$ ClinOncol 29: 215.

5. Citrin DL, Mortensen SJ, Grutsch JF, Neelam R (2013) Lack of patient compliance with adjuvant therapy guidelines is an important risk factor for recurrence in early breast cancer. World J of Psycho-Social Oncol 2: 1 .

6. Breast Cancer Survival Rates by Stage (2016) America Cancer Society.

7. Patient and Caregiver Resources, Nausea and Vomiting (2016) National Comprehensive Cancer Network.

8. Poole K (1996) The evolving role of the clinical nurse specialist within the comprehensive breast cancer centre. J ClinNurs 5: 341-349.[Crossref]

9. Landro L (2010) Taking Medical Jardon Out of Doctor Visits.The Wall Street Journal.

10. Martin LR, Williams SL, Haskard KB, Dimatteo MR (2005) The challenge of patient adherence. TherClin Risk Manag 1: 189-199.[Crossref]

11. Citrin DL (2014) Knowledge is Power. What every woman should know about breas cancer. Amazon Books.

12. Riffkin R (2014) Americans Rate Nurses Highest on Honesty. Ethical Standards Gallop.

Copyright: (C2016 Citrin DL. This is an open-access article distributed under the terms of the Creative Commons Attribution License, which permits unrestricted use, distribution, and reproduction in any medium, provided the original author and source are credited. 